\title{
Sufficient Conditions for Monotone Hazard Rate An Application to Latency-Probability Curves
}

\author{
Ewart A. C. Thomas
}

The University of Michigan, Ann Arbor, Michigan 48104

\begin{abstract}
It is assumed that when a subject makes a response after comparing a random variable with a fixed criterion, his response latency is inversely related to the distance between the value of the variable and the criterion. This paper then examines the relationship to response probability of (a) response latency, (b) difference between two latencies and (c) dispersion of latency, and also some properties of the latency distribution. It is shown that the latency-probability curve is decreasing if and only if the hazard function of the underlying distribution is increasing and, by using a fundamental lemma, sufficient conditions are obtained for monotone hazard rate.

An inequality is established which is satisfied by the slope of the Receiver Operating Characteristic under these sufficient conditions. Finally, a Latency Operating Characteristic is defined and it is suggested that such a plot can be useful in assessing the consistency between latency data and theory.
\end{abstract}

\section{INTRODUCTION}

Many authors have remarked that an analysis of the response times from a signal detection experiment can yield valuable information about the nature of the detection process (e.g. Carterette, 1966; Green and Luce, 1967; Laming, 1969). The method of such an analysis depends on the assumed detection and response time models, and on the response time statistic being observed, though the dependence on the former is more fundamental than that on the latter. The fact that detection models to be found in the above studies have at least one feature in common, namely, the random vairable which determines what response is made, is explicitly regarded as the outcome of a temporal process. Therefore, reaction time models can be derived from these detection models in a very natural way. For instance, in the model studied by Carterette (1966) the decision variable at time $t$ is $N(t)$, the reading on a hypothetical counter. A response time model is derived naturally if it is assumed that a response is made when $N(t)$ equals some critical value for the first time. 'I'he response time distribution would then be the first-passage time distribution of $N(t)$ through the critical value (that Carterette does not use the first-passage time distribution is one reason why his approach is difficult to follow).

(C) 1971 by Academic Press, Inc. 
The detection model to be assumed in this paper is similar to that of Signal Detection Theory (see Green and Swets, 1966). It is assumed that $X$ is a random variable which summarizes all of the information available to a subject on each (discrete) trial of a yes-no experiment ( $X$ is not necessarily a likelihood ratio.) The subject responds 'Yes' only if $X$ exceeds a predetermined criterion $c$, and responds 'No' otherwise. For our purposes, $X$ is regarded not as the outcome but as the starting-point of a temporal process, that of determining whether or bot $X$ exceeds $c$. Such a process has been considered by Smith (1968) and is roughly as follows:

(a) In order to determine whether $X$ exceeds $c$, the required precision in determining the value $x$ of $X$ is greater if $x$ is close to $c$ than if $x$ is very different from $c$.

(b) Precision is directly related to the number of fundamental operations.

(c) On the average, each fundamental operation lasts the same time.

It follows from (a)-(c) that response time or latency is inversely related to $|x-c|$. The derivation of this latency model from the detection model is not as natural as in the previously mentioned studies because $X$ is treated here as a 'single sample' rather than as the outcome of 'repeated sampling'.

Another factor influencing the present analysis is the latency statistic being studied. Some of the statistics that have been proposed are the latency operating characteristic curve (Carterette, Friedman and Cosmides, 1965), the tail of the latency distribution (Green and Luce, 1967) and the divisibility of the latency distribution (Laming, 1969). We will consider mainly the relationship between a location parameter of a response time distribution, e.g., the median, and the probability of the associated response. The use of quantiles rather than moments is due to two resaons. Firstly, many of the observed latency distributions have 'high' tails, suggesting that the median latency is a more reliable measure of location than the average latency. This is because of the possibility that if the empirical distribution were fitted by a theoretical 'high' tail distribution, this latter distribution may not have finite moments of any order. Secondly, to derive an expression for mean latency it would be necessary to know the functional dependence of latency on $|x-c|$. However, Andley and Mercer (1968) show that the use of median latency removes this difficulty. Let $m_{c}$ be such that, given noise alone, $\operatorname{Prob}\left(X \geqslant m_{c}\right)=1 / 2 \operatorname{Prob}(X \geqslant c)$, and let $t_{c}$ be the latency associated with an $X$ value of $m_{c}$. Since latency is a decreasing function of $|X-c|$, an observed latency would be less than $t_{c}$ if and only if the associated $X$ value exceeded $m_{c}$. Further, given noise alone, $X$ exceeds $m_{c}$ on half of the occasions that it exceeds $c$; therefore, observed latency is less than $t_{c}$ on half of the occasions when a 'Yes' response was given. In other words, $t_{c}$ is the median false alarm latency.

Although the use of quantiles allows us to ignore the two problems mentioned in the above paragraph, a third question has to be answered, namely, whether the latency quantiles are well-defined. This question will be considered in the next 
section. What is needed is a set of sufficient conditions for $t_{c}$ to be uniquely related to $m_{c}$, for then questions involving $t_{c}$ could be replaced by questions involving $m_{c}$.

Rather surprisingly, the relationship between $t_{c}$ and $\operatorname{Prob}(X \geqslant c)$ depends on the hazard function of the distribution of $X$. The following is a heuristic argument which suggests the nature of the dependence. Let $f(x)$ be the probability density function of $X$, and let $R(x)=\operatorname{Prob}(X \geqslant x)$; then $f(x) / R(x) \equiv r(x)$ is the hazard function. For a fixed small element $\Delta$, the probability that $c \leqslant X \leqslant c+\Delta$, given $c \leqslant X$ is approximately $r(c) \Delta$. Now there exists a latency $t(\Delta)$, depending on $\Delta$ but not on $c$, such that for $c \leqslant X \leqslant c+\Delta$ the associated latencies all exceed $t(\Delta)$. In other words, the proportion of the latency distribution to the right of the constant $t(\Delta)$ is directly proportional to $r(c)$, which suggests that the 'center' of the latency distribution increases with $c$ of $r(c)$ increases with $c$.

We now formally establish the relevance of the hazard function, and then we will provide sufficient conditions for it to be monotonic. Although the properties of distributions with monotone hazard rate have been extensively studied by Barlow, Marshall and Proschan (1963), and examples of distributions with nonmonotone hazard rate have been given by Thomas (1967), the sufficient conditions for monotonicity have not been stated before.

\section{Slope of the Latency-Probability Curve}

\section{Relevance of the Hazard Function}

Let $f(x)$ and $F(x)=1-R(x)$ be the probability density function (p.d.f.) and cumulative distribution function of $X$, respectively, and let $\lambda(|x-c|)$ be the value of the observed latency $T$, when $X=x$ and $c$ is the cut-off. We make the following two assumptions:

A2: $\quad R(x)$ is a strictly decreasing function of $x,{ }^{1}$

A2: $\lambda(x)$ is a strictly decreasing function of $x$ for $x>0$.

Al ensures that the quantiles of $f(x)$ are well defined.

In order to generalize the previous discussion on median latency, we define $m_{e, \alpha}$, for $0<\alpha \leqslant 1$, by the equation

$$
R\left(m_{c, \alpha}\right)=\alpha R(c)
$$

So that $m_{c, \alpha}$ is the quantile of order $(1-\alpha)$ of the distribution of $X$, given $X \geqslant c$; the p.d.f. of this conditional distribution is $f(x) / R(c), x \geqslant c$.

$$
\text { Let } t_{c, \alpha}=\lambda\left(m_{c, \alpha}-c\right) \text {. }
$$

${ }_{1}^{1}$ Throughout this paper it should be assumed that $x$ takes on values only in the range of $X$. 
Then, from A2,

$$
\operatorname{Prob}\left(T \leqslant t_{c, \alpha}\right)=\operatorname{Prob}\left(X>m_{c, \alpha}\right)=\alpha,
$$

that is, $t_{e, \alpha}$ is the quantile of order $\alpha$ of the latency distribution. Therefore, AI and A2 ensure that the latency quantiles and the quantiles of $f(x)$ are well defined and are in a $1-1$ correspondence with each other. The relationship between $t_{c, x}$ (location parameter) and $R(c)$ (response probability) can now be expressed as a relationship between $m_{c, x}$ and $c$ as follows:

LEMMA 2.1. $d t_{e, \alpha} / d R(c) \leqslant 0$ if and only if $d m_{c, \alpha} / d c \leqslant 1$.

Proof. By Al, $d R(c) / d c<0$, implying $d c / d R(c)<0$, and by $\mathrm{A} 2$ and (2),

$$
\frac{d t_{c, \alpha}}{d\left(m_{c, \alpha}-c\right)}<0 .
$$

Therefore, multiplying the preceding two inequalities,

$$
0<\frac{d c}{d R(c)} \cdot \frac{d t_{c, \alpha}}{d\left(m_{c, \alpha}-c\right)}=\frac{d c}{d\left(m_{c, \alpha}-c\right)} \cdot \frac{d t_{c, \alpha}}{d R(c)},
$$

from which we obtain

$$
\begin{array}{r}
\frac{d t_{c, \alpha}}{d R(c)} \leqslant 0 \text { if and only if } \frac{d\left(m_{c, \alpha}-c\right)}{d c} \leqslant 0 \\
\text { i.e. if and only if } \frac{d m_{c, \alpha}}{d c} \leqslant 1 .
\end{array}
$$

It can be readily verified that $d m_{c, \alpha} / d c$ is not necessarily less than 1 , even if $f(x)$ is symmetric about some $x$ value. To see this, we consider the Cauchy distribution with p.d.f. $f(x)=\left[\pi\left(1+x^{2}\right)\right]^{-1}$, which has been suggested by Laming (personal communication) as a possible model for the distribution of $X$. [Laming's reason for suggesting this distribution is that the convolution of any two distributions $f\left(x ; \mu_{1} \sigma_{1}\right), f\left(x ; \mu_{2}, \sigma_{2}\right)$ of the family $f(x ; \mu, \sigma)=\left\{\sigma \pi\left[1+((x-\mu) / \sigma)^{2}\right]\right\}^{-1}$ is $f\left(x ; \mu_{1}+\mu_{2}, \sigma_{1}+\sigma_{2}\right)$ which is also a member of the fanily.] For this distribution $m_{0, .5}=1$ and $m_{.1016} .5=1.1067$, so that $d m_{c, .5} / d c$ is greater than 1 when $c=0$. However, $d m_{c, \alpha} / d c$ is related to the hazard function.

THEOREM 2.2. $d m_{c, \alpha} / d c \leqslant 1$ for all $c$ and $\alpha$ if and only if the hazard function $f(x) / R(x)$ is increasing.

Proof. From (1) $m_{c, \alpha} \geqslant c$ and on differentiation,

$$
\frac{d m_{c, \alpha}}{d c}=\alpha \frac{f(c)}{f\left(m_{c, \alpha}\right)}=\frac{f(c)}{R(c)} \frac{R\left(m_{c, \alpha}\right)}{f\left(m_{c, \alpha}\right)} .
$$


Therefore, if $f(x) / R(x)$ is increasing,

$$
\frac{f(c)}{R(c)} \leqslant \frac{f\left(m_{c, \alpha}\right)}{R\left(m_{c, \alpha}\right)}
$$

which implies that $d m_{c, \alpha} / d c \leqslant 1$.

Suppose there exist $x^{\prime}$ and $x^{\prime \prime}\left(>x^{\prime}\right)$ such that

$$
\frac{f\left(x^{\prime}\right)}{R\left(x^{\prime}\right)}>\frac{f\left(x^{\prime \prime}\right)}{R\left(x^{\prime \prime}\right)} .
$$

Choose $\alpha^{\prime}=R\left(x^{\prime \prime}\right) / R\left(x^{\prime}\right)$. Then

$$
\left.\frac{d m_{e, \alpha^{\prime}}}{d c}\right|_{c=x^{\prime}}=\frac{f\left(x^{\prime}\right)}{R\left(x^{\prime}\right)} \frac{R\left(x^{\prime \prime}\right)}{f\left(x^{\prime \prime}\right)}>1,
$$

which completes the proof of the theorem.

Q.E.D.

Having established the relevance of the hazard function to the slope of the latencyprobability curve, we now provide sufficient conditions for it to be monotonic. The next section contains mathematical details and the reader interested only in the relevance of these details to data fitting may wish to omit it.

\section{Sufficient Conditions for Monotone Hazard Rate}

A fundamental lemma is needed.

Lemma 2.3. If $g(x)$ is an increasing (decreasing) function of $x$, then $E[g(X) \mid a \leqslant X \leqslant b]$ is an increasing (decreasing) function of $a$ and of $b$.

Proof.

$$
\begin{gathered}
\mu(a, b)=E[g(X) \mid a \leqslant X \leqslant b]=\frac{\int_{a}^{b} g(x) f(x) d x}{R(a)-R(b)}, \\
\frac{\partial \mu(a, b)}{\partial a}=\frac{f(a)\left\{\int_{a}^{b} f(x)[g(x)-g(a)] d x\right\}}{[R(a)-R(b)]^{2}}
\end{gathered}
$$

$\geqslant(\leqslant) 0$ if $g(x)$ is increasing (decreasing); and similarly for $\partial \mu(a, b) / \partial b$.

Q.E.D.

Two special cases of the lemma can be obtained by letting a tend to $-\infty$ for fixed $b$, and by letting $b$ tend to $\infty$ for fixed $a$.

It can now be seen that the hazard function is monotone if there exists a function $g(x)$ which is monotone and is such that

$$
f(x)=K \int_{n}^{\infty} g(y) f(y) d y,
$$


for then we would have

$$
\frac{f(x)}{R(x)}=K E[g(X), x \leqslant X],
$$

and a special case of Lemma 2.3 could be applied.

Theorem 2.4. If (i) $f(x)$ tends to 0 as $R(x)$ tends to 0 and (ii) $-\log f(x)$ is convex (concave), the hazard function is monotonically increasing (decreasing).

Note. If there is no finite $x^{\prime}$ such that $R\left(x^{\prime}\right)=0$, then (i) is automatically satisfied. If there is such an $x^{\prime}$ then (i) asserts that $\lim _{x \rightarrow x^{\prime}}-f(x)=0$.

Proof. Let $g(x)=-(d / d x) \log f(x)=-\left(f^{\prime}(x) / f(x)\right)$.

Then $g(x)$ is an increasing (decreasing) function if $\left(d^{2} / d x^{2}\right) \log f(x) \leqslant(\geqslant)$.

Also

$$
E[g(X) \mid a \leqslant X \leqslant b]=\frac{-\int_{a}^{b} \frac{f^{\prime}(x)}{f(x)} f(x) d x}{R(a)-R(b)}=\frac{f(a)-f(b)}{R(a)-R(b)} .
$$

Taking the limit $R(b) \rightarrow 0$, for fixed $a$, we have by condition (i)

$$
E[g(X) \mid a \leqslant X]=\frac{f(a)}{R(a)},
$$

the hazard function.

The result follows by an application of Lemma 2.3 .

Q.E.D.

Remark 2.5. Condition (ii) Theorem 2.4 is a necessary and sufficient condition for a family of p.d.f's, $f(x-\theta)$, to have monotone likelihood ratio in $x$ (Lehmann, 1959, p. 330). That is, if $x>x^{\prime}$ and $\theta>0$, then $f(x-\theta) / f(x)>f\left(x^{\prime}-\theta\right) / f\left(x^{\prime}\right)$ if and only if $-\log f$ is convex.

EXAMPLE 2.6. If $f(x)=(1 / \sqrt{2 \pi}) e^{-x^{2} / 2}$ (normal),

$$
\frac{d^{2}}{d x^{2}} \log f(x)=-1
$$

and if

$$
\begin{gathered}
f(x)=\frac{e^{-x}}{\left(1+e^{-x}\right)^{2}} \quad \text { (logistic), } \\
\frac{d^{2}}{d x^{2}} \log f(x)=-2 f(x),
\end{gathered}
$$

so that for both of these distributions the hazard function is increasing. 
Example 2.7. If condition (i) of Theorem 2.4 is violated, the conclusion is not necessarily true. Consider

$$
f(x)=\frac{1}{\log (1+g)} \frac{1}{(1+x)}, \quad 0 \leqslant x \leqslant g .
$$

Then $\left(d^{2} / d x^{2}\right) \log f(x)=(1+x)^{-2}>0$.

From $(1), m_{c, \alpha}=(1+c)^{\alpha}(1+g)^{1-\alpha}-1$,

which gives

$$
\frac{d m_{c, \alpha}}{d c}=\left[\frac{1+g}{1+c}\right]^{1-\alpha}, \quad 0 \leqslant c \leqslant g .
$$

Therefore, $d m_{c, \alpha} / d c \leqslant 1$ if $\log (1+g) \leqslant 1$, that is, if $g \leqslant e-1$. So that by Theorem 2.2, if $g<e-1$ the hazard function is monotonically increasing. If $g>e-1$,

$$
\begin{aligned}
\frac{d m_{c, \alpha}}{d c}>1 & \text { when } & c=0 \\
=\alpha \leqslant 1 & \text { when } & c=g,
\end{aligned}
$$

and in this case the hazard function is nonmonotonic.

ExAmple 2.8. The Cauchy distribution, $f(x)=\left[\pi\left(1+x^{2}\right)\right]^{-1}$, has been mentioned earlier when it was show that $d m_{c, .5} / d c$ is greater than $I$ when $c=0$. It follows from Theorems 2.2 and 2.4 that the hazard function for this distribution is not monotonically increasing; in fact, it is increasing for all $x$ less than 0.425 and decreasing for $x$ greater than this value. Also

$$
\frac{d^{2}}{d x^{2}} \log f(x)=\frac{2\left(x^{2}-1\right)}{\left(1+x^{2}\right)^{2}} \geqslant 0 \quad \text { if and only if } \quad|x| \geqslant 1 .
$$

Therefore, neither

$$
\left.\frac{d}{d x} \frac{f(x)}{R(x)}\right|_{x=x^{\prime}} \geqslant 0 \quad \text { nor }\left.\quad \frac{d^{2}}{d x^{2}} \log f(x)\right|_{x=x^{\prime}} \leqslant 0
$$

implies the other as is seen by taking $x^{\prime}=-1.1$ and 0.7 . However, a partial converse to Theorem 2.4 can be proved.

THEOREM 2.9. If (i) $f(x)$ tends to 0 as $R(x)$ tends to 0 , and (ii) $f(x) / R(x)$ is monotonically increasing (decreasing) for all $x$ greater than some finite value $x_{1}$, say, there exists a sequence $x_{1}<x_{2}<\cdots$ such that $\lim _{i \rightarrow \infty} R\left(x_{i}\right)=0$ and $\left\{u_{i}\right\}$ is monotonically increasing (decreasing), where

$$
u_{i}=-\left.\frac{d}{d x} \log f(x)\right|_{x=x_{i}}=-\frac{f^{\prime}\left(x_{i}\right)}{f\left(x_{i}\right)} .
$$


Proof. Since $f(x) \rightarrow 0$ as $R(x) \rightarrow 0$, by L'Hôpital's rule,

$$
\lim _{R(x) \rightarrow 0} \frac{f(x)}{R(x)}=-\lim _{R(x) \rightarrow 0} \frac{f^{\prime}(x)}{f(x)} .
$$

If

$$
0 \leqslant \frac{d}{d x} \frac{f(x)}{R(x)}=\frac{f^{\prime}(x) R(x)+f^{2}(x)}{R^{2}(x)}
$$

then

$$
\frac{f(x)}{R(x)} \geqslant-\frac{f^{\prime}(x)}{f(x)}
$$

Given $x_{i}$, let

$$
\delta_{i}=\frac{f\left(x_{i}\right)}{R\left(x_{i}\right)}-u_{i}
$$

By (4) there exists $x_{i}^{\prime}$ such that

$$
\frac{f(x)}{R(x)}+\frac{f^{\prime}(x)}{f(x)} \leqslant \delta_{i} \quad \text { for } \quad x \geqslant x_{i}^{\prime} .
$$

Choose $x_{i+1}>\max \left(x_{i}{ }^{\prime}, x_{i}\right)$. Then $x_{i+1}>x_{i}$ and

$$
\begin{aligned}
u_{i+1}=-\frac{f^{\prime}\left(x_{i+1}\right)}{f\left(x_{i+1}\right)} & \geqslant \frac{f\left(x_{i+1}\right)}{R\left(x_{i+1}\right)}-\delta_{i} \\
& =\frac{f\left(x_{i+1}\right)}{R\left(x_{i+1}\right)}-\frac{f\left(x_{i}\right)}{R\left(x_{i}\right)}+u_{i} \\
& \geqslant u_{i}, \text { since } \frac{f(x)}{R(x)} \text { is increasing. }
\end{aligned}
$$

The proof for decreasing hazard rate is similar.

Q.E.D.

EXAMPLE 2.10. In order to construct p.d.f's with monotone hazard rate, one can start with any monotone function $g(x)$, and then use (3). For example, if

$$
g(x)=\frac{x}{1+x}, \quad x \geqslant 0,
$$

then

$$
f(x)=(1 / 2)(1+x) e^{-x}
$$

For the p.d.f's to be symmteric, $g(x)$ has to be antisymmetric. 
Lemma 2.11. If $f(x)$ and $g(x)$ are related by (3), then $f(x)=f(-x)$ if and only if $g(x)=-g(-x)$.

Proof. Differentiating (3) yields

$$
g(x)=-\frac{f\left(x^{\prime}\right)}{K f(x)}
$$

If $f(x)=f(-x)$, then $f^{\prime}(x)=-f^{\prime}(-x)$, and from (5)

$$
g(x)=-g(-x) \text {. }
$$

Integrating (5) yields

$$
\log f(x)=-K \int g(x) d x
$$

Thus

$$
\begin{aligned}
\log f(-x) & =K \int g(-x) d x \\
& =-K \int g(x) d x \quad \text { if } \quad g(x)=-g(-x) \\
& =\log f(x) .
\end{aligned}
$$

Therefore $f(x)=f(-x)$.

\section{Application to Latency-Probability Curves}

The aim of the preceding results has been to provide simple sufficient conditions on $f(x)$ for the latency-probability curve to be monotonically decreasing. It was first shown that the curve is decreasing if and only if the hazard rate of $f(x)$ is increasing. Since the behavior of the hazard function is sometimes difficult to determine analytically (consider, for example, the normal distribution), simpler conditions were sought. These conditions were stated and discussed in the immediately preceding section, and the net result is the following theorem.

THEOREM 2.12. If $f(x)$ tends to 0 with $R(x)$, and if $-\log f(x)$ is convex, then the plot of response latency (quantile) against response probability is decreasing.

This result is strong because it is true for a latency quantile of any order. It is now simple to derive the weaker result for mean latency. Let us denote the latency p.d.f. by $p_{c}(t)$, and c.d.f. by $P_{c}(t)\left(p_{c}(t)\right.$ is a function of $f(x)$ and $\lambda(x)$, but it will not be necessary to make this dependence explicit). The quantiles $t_{c, \alpha}$, defined in (2), can also be defined by

$$
\alpha=\int_{0}^{t_{c, \alpha}} p_{c}(t) d t
$$


The mean latency $\tau_{c}$ is given by

$$
\tau_{c}=\int_{0}^{x} t p_{c}(t) d t=\int_{0}^{s}\left[1-P_{e}(t)\right] d t
$$

if the integral converges, and its dependence on $c$ is now stated.

THEOREM 2.132. If $f(x)$ tends to 0 with $R(x)$, and if $-\log f(x)$ is convex, then the plot of mean response latency against response probability is decreasing.

Proof. We have to show that $d t_{c, \alpha} / d c \leqslant 0,0<\alpha \leqslant 1$, implies that $d \tau_{c} / d c \leqslant 0$. Writing $1-\alpha$ for $\alpha$ in (7) we have

$$
\alpha=1-P_{e}\left(t_{c, 1-\alpha}\right)
$$

By referring to Fig. 1 it can be seen that

$$
\int_{0}^{x_{2}}\left[1-P_{c}(t)\right] d t=\int_{0}^{1} t_{c, 1-\alpha} d \alpha
$$

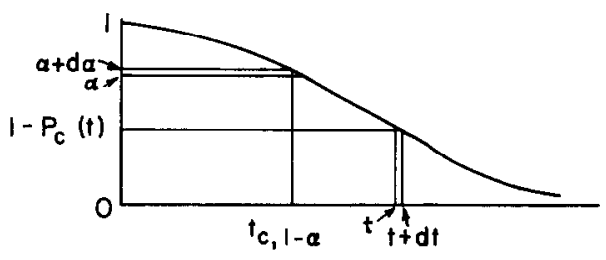

Figure 1

Therefore, from (8)

$$
\begin{aligned}
\frac{d \tau_{c}}{d c} & =\frac{d}{d c} \int_{0}^{\infty}\left[1-P_{c}(t)\right] d t=\frac{d}{d c} \int_{0}^{1} t_{c, 1-\alpha} d \alpha \\
& =\int_{0}^{1} \frac{\partial}{\partial c} t_{c, 1-\alpha} d \alpha
\end{aligned}
$$

if the integral converges,

$\leqslant 0$, since, by assumption, the integrand is negative.

We now consider the possibility that the slope of the latency-probability curve is constant. From (2) we have

$$
\frac{d t_{c, \alpha}}{d c}=\lambda^{\prime}\left(m_{c, \alpha}-c\right)\left(\frac{d m_{c, \alpha}}{d c}-1\right) .
$$

2 This theorem was first proved by Jerome L. Myers by a more direct method (personal communication). 
Therefore, the slope of the curve will not equal a positive constant unless $\lambda(x)$ and $f(x)$ are related in a very specific manner. For example, if $\lambda(x)=x^{-b}, b>0$, then the slope is constant only if $f(x)$ is such that

$$
m_{c, \alpha}=c+\left[b K_{\alpha}\left(c+g_{\alpha}\right)\right]^{-1 / b},
$$

where $K_{\alpha}$ and $g_{\alpha}$ are positive constants. Such a specific relationship between $\lambda(x)$ and $f(x)$ is very unlikely, so that strictly decreasing linear latency-probability curves are also unlikely.

However, it can be seen from (9) and A2 that latency is independent of response probability if and only if $d m_{c, \alpha} / d c$ equals 1 , and this motivates the following theorem.

TheоReм 2.14. Response latency is independent of response probability if and only if $f(x)$ is exponential.

Proof. We prove the stronger result that the following statements are equivalent:

$$
\begin{aligned}
\frac{d m_{c, \alpha}}{d c} & =K_{\alpha}, \quad \text { where } & & K_{\alpha} \leqslant 1 . \\
\frac{d m_{c, \alpha}}{d c} & =1 \quad \text { for all } \alpha . & & \\
f(x) & =\mu e^{-\mu x} \mu>0, & & \geqslant 0 \\
& =0 & & <0,
\end{aligned}
$$

for a suitable choice of origin.

We prove this by showing that (i) implies (ii) implies (iii) implies (ii). Statement (i) implies that $m_{c, \alpha}=K_{\alpha} c+h_{\alpha}$.

From (1), $m_{c, \alpha} \geqslant c$, so that, since $K_{\alpha} \leqslant 1$, we have $h_{\alpha} \geqslant 0$. Also since $m_{c .1}=c, h_{1}=0$. Differentiating (1) we see that $d m_{c, \alpha} / d c \geqslant 0$, which implies that $K_{\alpha} \geqslant 0$.

There is no finite $c^{\prime}$ such that $\operatorname{Prob}\left(X \leqslant c^{\prime}\right)=1$, for otherwise (10) would be violated when $c=c^{\prime}$. From (1),

$$
R\left(K_{\alpha} c+h_{\alpha}\right)=\alpha R(c) .
$$

Putting $c=0, h_{\alpha}, \ldots, h_{\alpha}\left(K_{\alpha}^{n-1}+\cdots+1\right)$ in (11), we get

$$
\begin{aligned}
R\left(h_{\alpha}\right) & =\alpha R(0), \\
R\left[h_{\alpha}\left(K_{\alpha}+1\right)\right] & =\alpha^{2} R(0), \\
R\left[h_{\alpha}\left(K_{\alpha}^{n}+\cdots+1\right)\right] & =\gamma^{n+1} R(0) .
\end{aligned}
$$

If

$$
K_{\alpha}<1, \lim _{n \rightarrow \infty}\left(K_{\alpha}^{n}+\cdots+1\right)=\left(1-K_{\alpha}\right)^{-1},
$$


which implies that $\operatorname{Prob}\left[X \leqslant h_{\alpha}\left(1-K_{\alpha}\right)^{-1}\right]=1$, which is contradictory. Therefore, $K=1$, which is statement (ii).

Statement (ii) implies that

$$
m_{c, \alpha}=c+h_{\alpha}
$$

from which we get

$$
R\left(c+h_{\alpha}\right)=\alpha R(c)
$$

and

$$
f\left(c+h_{\alpha}\right)=\alpha f(c)
$$

There exists a finite number $a$ such that $\operatorname{Prob}(X \geqslant a)=1$, for otherwise we would have, on integrating (14) from $-\infty$ to $\infty$,

$$
1=\alpha \quad \text { for all } \alpha,
$$

which is absurd. Let $a=0$, which implies that

$$
f(c)=0, \quad c<0
$$

and let

$$
\left.\frac{d h_{\alpha}}{d \alpha}\right|_{\alpha=1}=\frac{1}{\mu}
$$

They, from (13),

$$
\frac{R\left(c+h_{\alpha}\right)-R(c)}{h_{\alpha}}=-\frac{(1-\alpha)}{h_{\alpha}} R(c) .
$$

Taking limits of both sides as $\alpha \cdots>1$, we get

$$
\begin{aligned}
\frac{d R(c)}{d c} & =-\mu R(c), \\
R(c) & =e^{-\mu c} .
\end{aligned}
$$

Therefore, recalling (15),

$$
\begin{aligned}
f(c) & =\mu e^{-\mu c}, & & c \geqslant 0 \\
& =0 & & c<0,
\end{aligned}
$$

which is statement (iii). Clearly, (iii) implies (ii). 
The exponential distribution is the only distribution for which $d^{2} / d x^{2} \log f(x)$ is zero, and the above theorem is one illustration of how special data predictions arise from its adoption. When we consider the second-order properties of the latencyprobability curve in the next section, we will encounter another such illustration.

\section{Second-Order Properties of Latency}

In the typical signal detection experiment there are four classes of latency corresponding to the four classes of responses, hits, false alarms, misses and correct rejections. The previous sections stated results concerning the rate of change of latency of one class, say false alarm, as the criterion $c$ is varied. We now consider the rate of change, as $c$ is varied, of

(a) the difference between 'typical' latencies from two classes, e.g., that between median false alarm and median hit latencies, and

(b) the difference between two latency quantiles from the same distribution, e.g., $\left(t_{c, \alpha}-t_{c, \alpha^{\prime}}\right), \alpha \neq \alpha^{\prime}$. A special case is the semiinterquartile range, which is a measure of dispersion of the latency distribution.

The difference between 'typical' hit and false alarm latencies is simply expressed if it is assumed that the distributions of $X$ given noise and signal plus noise have the same shape. Let $f(x)$ and $f(x-\mu), \mu>0$, be the distributions of $X$ given noise and signal plus noise, respectively. Then $t_{e, \alpha}$, as defined in (2), is the quantile of order $\alpha$ from the false alarm latency distribution, and the quantile of order $\alpha$ from the hit latency distribution is

$$
t_{e-\mu, \alpha}=\lambda\left(m_{c-\mu, \alpha}-c+\mu\right) .
$$

Therefore,

$$
\begin{aligned}
\frac{\partial}{\partial c}\left[t_{c, \alpha}-t_{c-\mu, \alpha}\right]= & \lambda^{\prime}(m-c)\left[\frac{\partial m_{c, \alpha}}{\partial c}-1\right] \\
& -\lambda^{\prime}\left(m_{c-\mu}-c+\mu\right)\left[\frac{\partial m_{c-\mu, \alpha}}{\partial c}-1\right] .
\end{aligned}
$$

One of the conditions influencing the sign of the right side of (17) is the sign of $\left[\partial m_{c, \alpha} / \partial c-\partial m_{c-\mu, \alpha} / \partial c\right]$, which is determined by the sign of $\partial^{2} m_{c, \alpha} / \partial c^{2}$.

It will be shown later that the sign of the rate of change $\left(t_{c, \alpha}-t_{c, \alpha}\right)$ with $c$ depends on, among other things, the sign of $\partial^{2} m_{c, \alpha} / \partial c \partial \alpha$. Thus a prerequisite for our discussion of the second order properties of latency is a study of the second derivatives of $m_{c, \alpha}$. The developement in the next section parallels that in section II. 
Second Partial Derivatives of $m_{c, \alpha}$.

Differentiating (1) we obtain

$$
\begin{aligned}
& \frac{\partial^{2} m_{e, \alpha}}{\partial \alpha^{2}}=-\frac{R^{2}(c)}{f^{2}\left(m_{c, \alpha}\right)} f^{\prime}\left(m_{c, \alpha}\right), \\
& \frac{\partial^{2} m_{c, \alpha}}{\partial c \partial \alpha}=\frac{f(c)}{f\left(m_{e, \alpha}\right)}\left[1+\frac{f^{\prime}\left(m_{e, \alpha}\right) R\left(m_{c, \alpha}\right)}{f^{2}\left(m_{e, \alpha}\right)}\right]
\end{aligned}
$$

and

$$
\frac{\partial^{2} m_{c, \alpha}}{\partial c^{2}}=\frac{\alpha f^{2}(c)}{f\left(m_{c, \alpha}\right) R(c)}\left[\frac{f^{\prime}(c) R(c)}{f^{2}(c)}-\frac{f^{\prime}\left(m_{c, \alpha}\right) R\left(m_{c, \alpha}\right)}{f^{2}\left(m_{c, \alpha}\right)}\right] .
$$

We now state some results concerning these derivatives.

THEOREM 3.1. $\partial^{2} m_{c, \alpha} / \partial \alpha^{2}$ has the same sign as $-f^{\prime}\left(m_{c, \alpha}\right)$. The proof of this is immediate.

THEOREM 3.2. $\partial^{2} m_{c, \alpha} / \partial c \partial \alpha \geqslant 0$ if and only if the hazard function is increasing.

Proof.

$$
\frac{d}{d x} \frac{f(x)}{R(x)}-\frac{f^{2}(x)}{R^{2}(x)}\left[1+\frac{f^{\prime}(x) R(x)}{f^{2}(x)}\right]
$$

which has the same sign as the right side of (19).

Q.E.D.

Corollary 3.3. If $f(x) \rightarrow 0$ as $R(x) \rightarrow 0$, and if $-\log f(x)$ is convex, then $\partial^{2} m_{c, \alpha} / \partial c \partial \alpha \geqslant 0$.

Proof. The proof follows from Theorems 2.4 and 3.2.

Q.E.D.

Theorem 3.4. If $-\log f(x)$ is convex and if $f^{\prime}(c) \geqslant 0$, then $\partial^{2} m_{c, \alpha} / \partial c^{2} \geqslant 0$.

Proof.

$$
\begin{aligned}
\frac{d^{2}}{d x^{2}}[-\log f(x)] & =\frac{f^{\prime 2}(x)-f(x) f^{\prime \prime}(x)}{f^{2}(x)} \\
\frac{d}{d x} \frac{f^{\prime}(x) R(x)}{f^{2}(x)} & -f^{-3}(x)\left\{R(x)\left[f(x) f^{\prime \prime}(x)-f^{\prime 2}(x)\right]-f^{\prime 2}(x) R(x)-f^{\prime}(x) f^{2}(x)\right\} \\
& \leqslant 0 \quad \text { if } f^{\prime}(x) \geqslant 0 \quad \text { and } \quad-\log f(x) \text { is convex. }
\end{aligned}
$$

Therefore, since $m_{c, \alpha} \geqslant c$, the right side of (20) is positive. Q.E.D.

THEOREM 3.5. If there exists a such that $f^{\prime}(x) \neq 0$ for $x>a$, and if $-f(x) / f^{\prime}(x)$ is convex for $x>a$, then $\partial^{2} m_{c, \alpha} / \partial c^{2} \geqslant 0$ for $c>a$. 
Proof. As in the immediately preceding proof, we have to show that $\left[f^{\prime}(x) R(x)\right] / f^{2}(x)$ is a decreasing function, or, equivalently, that $\left[f^{2}(x) / f^{\prime}(x)\right] / R(x)$ is an increasing function for $x>a$. Lemma 2.3 (with $b$ infinite) would be applicable if it can be shown that

$$
\frac{f^{2}(x) / f^{\prime}(x)}{R(x)}=E[g(X) \mid x \leqslant X],
$$

where $g(x)$ is an increasing function.

If

$$
\frac{f^{2}(x)}{f^{\prime}(x)}=\int_{x}^{\infty} g(y) f(y) d y
$$

then

$$
\begin{aligned}
g(x) & =\frac{f(x) f^{\prime \prime}(x)}{f^{\prime 2}(x)}-2 \\
& =\frac{d}{d x}\left[x-\frac{f(x)}{f^{\prime}(x)}\right]-2 \\
& =\frac{d}{d x}\left[-\frac{f(x)}{f^{\prime}(x)}\right]-1 .
\end{aligned}
$$

Therefore, $g(x)$ is increasing if $-f(x) / f^{\prime}(x)$ is convex, from which the result follows.

EXAMPLE 3.6. If $f(x)=(1 / \sqrt{2 \pi}) e^{-x^{2} / 2},-f(x) / f^{\prime}(x)=1 / x$, which is a convex function for $x>0$, and if

$$
\begin{aligned}
f(x) & =\frac{e^{-x}}{\left(1+e^{-x}\right)^{2}}, \\
-\frac{f(x)}{f^{\prime}(x)} & =1+\frac{2}{e^{x}-1},
\end{aligned}
$$

which is convex for $x>0$. Therefore, for both these distributions, $\partial^{2} m_{c, \alpha} / \partial c^{2} \geqslant 0$ for $c>0$, by Theorem 3.5. Also, by Theorem 3.4, $\partial^{2} m_{c, \alpha} / \partial c^{2} \geqslant 0$ for $c \leqslant 0$. Thus $\partial^{2} m_{e, \alpha} / \partial c^{2} \geqslant 0$ for all $c$.

THEOREM 3.7. The convexity of $-\log f(x), x>a$, does not imply the convexity of $-f(x) / f^{\prime}(x), x>a$, and vice versa.

Proof. We prove this result by providing two counter-examples:

(i) The Couchy distribution. It has been shown earlier that $-\log f(x)$ is concave for $x>1$. 
However,

$$
-\frac{f(x)}{f^{\prime}(x)}=\frac{1}{2}\left(x+x^{-1}\right)
$$

which is convex for $x>0$.

(ii) The Beta distribution. Let

$$
\begin{gathered}
f(x)=\frac{\Gamma\left(2 \frac{1}{2}\right)}{\Gamma\left(\frac{1}{2}\right)} x^{-1 / 2}(1-x), \quad 0<x<1 . \\
-\frac{d^{2}}{d x^{2}} \log f(x)=\frac{x^{2}+2 x-1}{2 x^{2}(1-x)^{2}}>0 \quad \text { for } \quad x>.42 .
\end{gathered}
$$

However, $-f(x) / f^{\prime}(x)=x-x^{2}$, which is concave.

The above theorem states that if $m_{c \cdot \alpha}-c$ is plotted as a function of $c$, the sign of the slope and the sign of the curvature are logically independent. However, it can be verified that the curvature is positive for most of the distributions commonly encountered in modeling.

As indicated earlier, the exponential distribution provides a limiting case.

Theorem 3.8. $\partial^{2} m_{c, \alpha} / \partial c^{2}=0$ for all $c$ if and only if

$$
f(x)=a_{1} e^{-a_{2} x}, \quad x \geqslant a_{3}
$$

or

$$
f(x)=b_{1} x^{b_{2}}, \quad x \geqslant b_{3}
$$

for suitable $a_{1}, a_{2}, a_{3}, b_{1}, b_{2}$ and $b_{3}$.

Proof. $d / d x\left[f^{\prime}(x) R(x)\right] / f^{2}(x)=0$ if and only if $R^{K}(x) f(x)=h$, where $K$ and $h$ are constants. When $K=-1, f(x)$ is exponential; otherwise $f(x)$ is proportional to a power of $x$.

Q.E.D.

\section{Application to Latency Curves.}

In order to determine the slope of the latency-probability curve in Section 2, we had to specify two sets of conditions. The first set was of conditions on $f(x)$ such that the first derivative of $\left(m_{e, \alpha}-c\right)$ was negative, and the second condition was that the slope of $\lambda(x)$ was negative. Similarly, to determine the second-order properties of latency we need to consider the second derivative of $\left(m_{c, \alpha}-c\right)$ and the curvature of $\lambda(x)$. In the preceding subsection, sufficient conditions were given for $\partial^{2} m_{e, \alpha} / \partial c^{2}$ to be positive. We can now use (17) to prove the following theorem. 
THEOREM 3.9. If there exists a such that

(i) $\begin{array}{rlrl}f^{\prime}(x) & \geqslant 0 & \text { for } & x \leqslant a \\ <0 & & x>a,\end{array}$

(ii) $f(x)$ tends to 0 as $R(x)$ tends to 0 ,

(iii) $-\log f(x)$ is convex for all $x$, and

(iv) $\frac{-f(x)}{f^{\prime}(x)}$ is convex for $x>a$,

then if $t_{c, \alpha}-t_{c-\mu, \alpha}$ increases with $t_{c, \alpha}$ for fixed $\alpha$ and $\mu(>0), \lambda(x)$ is convex.

Proof. Conditions (ii) and (iii) ensure that $\partial m_{c, \alpha} / \partial c \leqslant 1$ and $\partial t_{c, \alpha} / \partial c \geqslant 0$ (Theorem 2.4), and (i), (ii) and (iv) ensure that $\partial^{2} m_{c, \alpha} / \partial c^{2} \geqslant 0$ (Theorems 3.4 and 3.5). Since $\partial^{2} m_{c, \alpha} / \partial c^{2} \geqslant 0,0 \geqslant \partial m_{c, \alpha} / \partial c-1 \geqslant \partial m_{c-\mu, \alpha} / \partial c-1$.

Therefore, the right side of (17) can be positive only if

$$
\left|\lambda^{\prime}\left(m_{c, \alpha}-c\right)\left[\frac{\partial m_{c, \alpha}}{\partial c}-1\right]\right| \geqslant\left|\lambda^{\prime}\left(m_{c-\mu, \alpha}-c+\mu\right)\left[\frac{\partial m_{c-\mu, \alpha}}{\partial c}-1\right]\right| .
$$

Since $\lambda^{\prime}(x)<0$, this holds only if

$$
\left|\lambda^{\prime}\left(m_{c, \alpha}-c\right)\right|>\left|\lambda^{\prime}\left(m_{c-\mu, \alpha}-c+\mu\right)\right| .
$$

Now $0<m_{c, \alpha}-c<m_{c-\mu, \alpha}-c+\mu$ since $\partial m_{c, \alpha} / \partial c \leqslant 1$; thereforc (21) is the condition for $\lambda(x)$ to be convex.

Q.E.D.

Application to Dispersion of Latency.

In many experimentalstudies involving reaction time measurements it has been found that dispersion of reaction time increases as 'typical' (e.g., median) reaction time increases. The following results determine the conditions on $\lambda(x)$ under which dispersion and location of latency are directly related.

THEOREM 3.10. If (i) $f(x)$ tends to 0 as $R(x)$ tends to 0 , (ii) $-\log f(x)$ is convex and (iii) $\lambda(x)$ is concave, then $t_{c, \alpha}-t_{c, \alpha^{\prime}}, \alpha>\alpha^{\prime}$, decreases as $t_{c, \alpha}$ as $t_{c, \alpha}$ increases; i.e., $\partial^{2} t_{e, \alpha} / \partial c \partial \alpha \leqslant 0$.

Proof. Conditions (i) and (ii) ensure that $\partial m_{c, \alpha} / \partial c \leqslant 1, \partial t_{c, \alpha} / \partial c \geqslant 0$ (Theorem 2.4), and that $\partial^{2} m_{c, \alpha} / \partial c \partial \alpha \geqslant 0$ (Theorem 3.2). Let $c>c^{\prime}, \alpha>\alpha^{\prime}$ and let $Z_{c, \alpha}=m_{c, \alpha}-c$. Now

$$
\begin{aligned}
Z_{c, \alpha^{\prime}} & >Z_{c, \alpha}, \\
Z_{c^{\prime}, \alpha} & >Z_{c, \alpha}, \quad \text { since } \quad \frac{\partial m_{c, \alpha}}{\partial c} \leqslant 1, \quad \text { and } \\
0 & <Z_{c^{\prime}, \alpha}-Z_{c, \alpha}<Z_{c^{\prime}, \alpha^{\prime}}-Z_{c, \alpha^{\prime}}, \quad \text { since } \quad \frac{\partial^{2} m_{c, \alpha}}{\partial c \partial \alpha} \geqslant 0 .
\end{aligned}
$$


Since $Z_{c, x^{\prime}}>Z_{c, a}$, if $\lambda(x)$ is concave,

$$
0<\lambda\left(Z_{c, \alpha}\right)-\lambda\left(Z_{c^{\prime}, \alpha}\right)<\lambda\left(Z_{c^{\prime}, \alpha^{\prime}}\right)-\lambda\left(Z_{c^{\prime}, \Omega^{\prime}}\right),
$$

i.e.,

$$
0<t_{e, \alpha}-t_{c^{\prime} x}<t_{c, \alpha^{\prime}}-t_{c^{\prime}, \alpha^{\prime}},
$$

i.e.,

$$
0<t_{c, \alpha}-t_{c, \alpha^{\prime}}<t_{c^{\prime}, \alpha}-t_{c^{\prime}, \alpha^{\prime}}
$$

i.e.,

$$
\frac{\partial^{2} t_{c, \alpha}}{\partial c \partial \alpha} \leqslant 0
$$

Another form of this result is

THEOREM 3.10(a). If the dispersion and location of (false-alarm) latencies are directly related, then $\lambda(x)$ is convex.

We now prove that the dispersion measure $t_{c, \alpha}-t_{c, \alpha^{\prime}}$ is directly related to the variance of latency. The following lemma is needed concerning $p_{c}(t)$, the $\mathrm{p} \cdot \mathrm{d} \cdot \mathrm{f}$ of latency.

Lemma 3.11. If (i) $\partial t_{e, \alpha} / \partial c \geqslant 0$ and (ii) $\partial^{2} t_{c, \alpha} / \partial c \partial \alpha \geqslant 0$, then $\partial / \partial c p_{c}\left[P_{c}^{-1}(\alpha)\right] \leqslant 0$.

Proof. Let $c>c^{\prime}$ and $\alpha>\alpha^{\prime}$. Then since $\partial t_{c, \alpha} / \partial c \geqslant 0 . P_{c}(t) \leqslant P_{c^{\prime}}(t)$. Since $\partial^{2} t_{c, \alpha} / \partial c \partial \alpha \geqslant 0,0<t_{c, \alpha^{\prime}}-t_{c^{\prime}, \alpha^{\prime}}<t_{c, \alpha}-t_{c^{\prime}, \alpha}$.

Letting $\alpha^{\prime} \rightarrow \alpha$, the last inequality implies that

$$
p_{c^{\prime}}\left(t_{c^{\prime}, \alpha}\right) \geqslant p_{c}\left(t_{c, \alpha}\right),
$$

which, since $P_{c}\left(t_{c, \alpha}\right)=\alpha$, implies

$$
p_{c^{\prime}}\left[P_{c^{\prime}}^{-1}(\alpha)\right] \geqslant p_{c}\left[P_{c}^{-1}(\alpha)\right]
$$

i.e.,

$$
\frac{\partial}{\partial c} p_{c}\left[P_{c}^{-1}(\alpha)\right] \leqslant 0
$$

Theorem 3.12. The dispersion $t_{c, \alpha}-t_{c, \alpha^{\prime}}\left(\alpha>\alpha^{\prime}\right)$ and variance of latency are directly related. 
Proof. We let $\sigma_{c}{ }^{2}$ denote the variance of latency. We have to show that if $\partial t_{c, \alpha} / \partial c$ and $\partial^{2} t_{c, \alpha} / \partial c \partial \alpha$ are both positive, $\sigma_{c}^{2}$ increases with $c$.

$$
\begin{aligned}
\sigma_{c}^{2} & =\int_{0}^{\infty}\left(t-\tau_{c}\right)^{2} p_{c}(t) d t, \quad \text { where } \quad \tau_{c}-\int_{0}^{\infty} t p_{c}(t) d t \\
& =\int_{0}^{\infty} t^{2} p_{r}(t) d t-\left[\int_{0}^{\infty}\left[1-P_{o}(t)\right] d t\right]^{2}
\end{aligned}
$$

Now

$$
\int_{0}^{\infty} t^{2} p_{c}(t) d t=2 \int_{0}^{\infty} t\left[1-P_{c}(t)\right] d t=2 \int_{0}^{\infty}\left[1-P_{c}(t)\right] \int_{0}^{t} d s d t
$$

and

$$
\begin{aligned}
{\left[\int_{0}^{\infty}\left[1-P_{c}(t)\right] d t\right]^{2} } & =\int_{0}^{\infty}\left[1-P_{c}(t)\right] d t \int_{0}^{\infty}\left[1-P_{c}(s)\right] d s \\
& =2 \int_{0}^{\infty}\left[1-P_{c}(t)\right] \int_{0}^{t}\left[1-P_{c}(s)\right] d s d t
\end{aligned}
$$

Therefore,

$$
\sigma_{c}^{2}=2\left\{\int_{0}^{\infty}\left[1-P_{c}(t)\right] \int_{0}^{t} P_{c}(s) d s d t\right\}
$$

Let $u=P_{c}(t), v=P_{c}(s)$. Then

$$
d t=\frac{d u}{p_{c}\left[P_{c}^{-1}(u)\right]} \quad \text { and } \quad d s=\frac{d v}{p_{c}\left[P_{c}^{-1}(v)\right]} .
$$

Therefore,

$$
\sigma_{c}^{2}=2\left\{\int_{0}^{1}(1-u) \int_{0}^{u} \frac{v d v}{p_{c}\left[P_{c}^{-1}(v)\right]} \frac{d u}{p_{c}\left[P_{c}^{-1}(u)\right]}\right\}
$$

By Lemma 3.11, the derivative of the integrand with respect to $c$ is positive. Therefore, if the integral converges,

$$
\frac{d}{d c} \sigma_{c}^{2} \geqslant 0
$$

\section{Extension to Negative Responses}

All of the analysis so far has been concerned with the latency of false alarms and hits, which occur when the value of $X$ exceeds the criterion $c$. The analysis of negative response latencies, i.e., miss and correct rejection latencies, is similar to that of positive responses. 
Let $M_{c, \alpha}$ be defined by

$$
F\left(M_{c . \alpha}\right)=\alpha \cdot \alpha(c)
$$

and let

$$
T_{c, \alpha}=\lambda\left(c-M_{c, \alpha}\right)
$$

Then $T_{c, \alpha}$ and $T_{c-\mu, \alpha}$ are the quantiles of order $\alpha$ of the correct rejection and miss latency distributions, respectively.

Applying Lemma 2.3 (with $a \rightarrow-\infty$ ) it can be shown that

(i) if $-\log f(x)$ is convex, then $f(x) / F(x)$ is decreasing (cf. Theorem 2.4) and

(ii) if $f(x) / f^{\prime}(x)$ is convex for $x \leqslant a,\left[f^{\prime}(x) F(x)\right] / f^{2}(x)$ is decreasing for $x \leqslant a$ (cf. Theorem 3.5).

Hence, by differentiating (22) it can be shown that

$$
\begin{array}{r}
\frac{\partial}{\partial c}\left(c-M_{c, \alpha}\right) \geqslant 0 \\
\frac{\partial^{2}}{\partial c \partial \alpha}\left(c-M_{c, \alpha}\right) \leqslant 0
\end{array}
$$

and

$$
\frac{\partial^{2}}{\partial c^{2}}\left(c-M_{c . \alpha}\right) \geqslant 0
$$

from which it follows that

(a) if $T_{c-\mu, \alpha}-T_{c, \alpha}$ decreases as $T_{c, \alpha}$ decreases, $\lambda(x)$ is convex (cf. Theorem 3.9),

(b) if the dispersion and location of (correct rejection) latency are directly related then $\lambda(x)$ is convex (cf. Theorem $3.10(a)$ ), and

(c) the dispersion, $T_{c, \alpha} \quad T_{c, \alpha^{\prime}}$, and the corresponding variance are directly related (cf. Theorem 3.12).

\section{IV, Some Properties of The Latency Distribution}

It was indicated in Section 2 that it was necessary to assume that $\lambda(x)$ is strictly decreasing in order to ensure that the latency quantiles were well-defined. One way of seeing this is to suppose that

and

$$
\lambda(x)=0 \quad x \geqslant x_{0},
$$

$$
\operatorname{Prob}\left(X>x_{0}\right)>0 .
$$


Then, denoting latency by $T$,

$$
\operatorname{Prob}(T=0)=\operatorname{Prob}\left(X>x_{0}\right)>0,
$$

so that $p_{c}(t)$ would have a discrete mass at the origin. Therefore, A2 ensures that $p_{c}(t)$ has no discrete masses.

Another desirable property of $p_{c}(t)$ is that $P_{c}(t)$ should be less than 1 for all finite $t$, i.e., the range of $T$ should be semiinfinite. If $\lambda_{0}=\lim _{x \rightarrow 0} \lambda(x)$ is finite, then $\operatorname{Prob}\left(T>\lambda_{0}\right)$ would be zero, so that a necessary and sufficient condition for the range of $T$ to be semifinite is that

$$
\lim _{x \rightarrow 0} \lambda(x)=\infty
$$

A third property of latency distributions is the empirical one that they have 'high' tails. A useful measure for deciding whether or not a distribution has a high tail is the convexity of $-\log p_{c}(t)$, a measurc which has been central to the analysis in this paper. To see the relevance of $-\log p_{c}(t)$, suppose that, for $n>0$ and large $t$,

$$
p_{c}(t) \sim A t^{-n} .
$$

Then

$$
-\log p_{c}(t) \sim-\log A+n \log t
$$

and

$$
\frac{d^{2}}{d t^{2}}\left[-\log p_{c}(t)\right] \sim-\frac{n}{t^{2}}<0
$$

Now suppose that

$$
p_{c}(t) \sim A e^{-t^{n}}
$$

Then

$$
-\log p_{c}(t) \sim-\log A+t^{n}
$$

and

$$
d t^{2}\left[-\log p_{\varepsilon}(t)\right] \sim n(n-1) t^{n-2} \geqslant 0 \quad \text { if } \quad n \geqslant 1 .
$$

Distributions of the form (25) are said to have a high tail, whereas the exponential types in $(26)(n \geqslant 1)$ are said to have a low tail. The above argument shows that a sensible definition of a low tailed latency distribution is one for which $-\log p_{c}(t)$ is convex.

We now investigate the convexity of $-\log p_{c}(t)$ for simple choices of $\lambda(x)$ and $f(x)$. 
The point we hope to make is that it is "easier" to derive a model for a high tailed latency distribution than a low tailed one. Now

$$
\begin{aligned}
P_{c}(t) & =\operatorname{Prob}(T \leqslant t) \\
& =\operatorname{Prob}\left[X-c>\lambda^{-1}(t) \mid X>c\right] \\
& =\frac{R\left[c+\lambda^{-1}(t)\right]}{R(c)} .
\end{aligned}
$$

Therefore,

$$
p_{c}(t)=\frac{1}{\mid \lambda^{\prime}(x) !} \frac{f\left[c+\lambda^{-1}(t)\right]}{R(c)} .
$$

EXAMPLES 4.1.

$$
\begin{gathered}
\lambda(x)=x^{-b}, \quad b>0, \quad x>0 . \\
p_{c}(t)-\frac{1}{t^{(1+b) / b}} \frac{f\left(c+t^{-1 / b}\right)}{R(c)} . \\
\frac{d^{2}}{d t^{2}} \log p_{c}(t) \alpha^{l}\left\{\frac{b(1+b)}{x^{2}}+\frac{(b+1)}{x} \frac{f^{\prime}(c+x)}{f(c+x)}+\frac{d^{2}}{d x^{2}} \log f(c+x)\right\},
\end{gathered}
$$

where $t=x^{-b}$. For most distributions commonly encountered,

$$
\lim _{x \rightarrow 0} \frac{f^{\prime}(c+x)}{f(c+x)}=O(1)
$$

and

$$
\lim _{x \rightarrow 0} \frac{d^{2}}{d x^{2}} \log f(c+x)=O(1) .
$$

'T'herefore, since $t \rightarrow \infty$ as $x \rightarrow O$, for large $t$

$$
\frac{d^{2}}{d t^{2}} \log p_{c}(t)=0\left(t^{2 / b}\right)
$$

i.e., $-\log p_{e}(t)$ is concave for large $t$ for these distributions.

Incidentally,

$$
\lim _{t \rightarrow 0} p_{c}(t)=0
$$

if

$$
\lim _{t \rightarrow 0} \frac{f\left(c+t^{-1 / b}\right)}{t^{(1+b) / b}}=0,
$$

i.e. if

$$
\lim _{x \rightarrow \infty} x^{b+1} f(c+x)=0,
$$

which is satisfied if $f(x)$ is of the type given in (26). 
If $\lambda(x)=x^{-1 / 2} e^{-x}$, it can be shown that for large $t$ and distributions satisfying (28) and (29),

$$
\frac{d^{2}}{d t^{2}} \log p_{c}(t)=O\left(t^{2}\right)
$$

so that $-\log p_{c}(t)$ is again concave.

We conclude this section by giving a necessary and sufficient condition for mean latency to exist.

Theоrem 4.2. If $x_{*}$ is such that, for $x_{*} \leqslant x, f(x)$ is strictly positive and bounded, then $p_{c}(t)$ has a finite mean for $x_{*} \leqslant c$ if and only if $\int_{0}^{\eta} \lambda(x) d x$ converges for arbitrary $\eta>0$.

Proof.

$$
\begin{aligned}
E(T) & =\frac{1}{R(c)} \int_{0}^{\infty} \lambda(x) f(c+x) d x \\
& =\frac{1}{R(c)}\left\{\int_{0}^{n} \lambda(x) f(c+x) d x+\int_{n}^{\infty} \lambda(x) f(c+x) d x\right\} .
\end{aligned}
$$

Let $f_{m}$ and $f_{M}$ be the minimum and maximum values, respectively, of $f(c+x)$ for $x \geqslant 0$. Then

$$
\frac{f_{m}}{R(c)} \int_{0}^{\eta} \lambda(x) d x<E(T)<\frac{1}{R(c)}\left\{f_{M} \int_{0}^{\eta} \lambda(x) d x+\lambda(\eta) R(c+\eta)\right\},
$$

from which the result follows.

Q.E.D.

Remark 4.3. If $\lambda(x)=x^{-b}$, mean latency exists if and only if $0<b<1$.

\section{Receiver and Latency Operating Characteristics}

The plotting of Operating Characteristics is often a useful way of comparing data with theoretical predictions. The Receiver Operating Characteristic (ROC) is the plot of the hit rate, $p_{H}(c)$, against the false alarm rate, $p_{F A}(c)$, as the criterion $c$ is varied. In view of the distinction which has been drawn between those distributions for which $-\log f(x)$ is convex and the remainder, it is of interest to discover whether the ROCs yielded by the two classes of distributions are different in any way.

There have been at least two types of Latency Operating Characteristics (LOC) proposed in the literature. One kind, proposed by Carterette, et al. (1965), is the plot of the proportion of hit latencies longer than $\gamma$ against the proportion of false alarm latencies longer than $\gamma$, as $\gamma$ varies from 0 to $\infty$. The other kind, proposed by Norman 
and Wicklegren (1969), Moss, Myers and Filmore (1970), Myers and Thomas (1970), is similar to the first kind, except that it plots unconditional frequencies of latencies and uses all four classes of latencies in a single plot. Both kinds of LOC can be plotted using the latency data from a single criterion setting, and the plotting procedure involves separating the latencies into four classes.

A third type of LOC is the plot of median latency, given that the signal plus noise was presented, against median latency, given that noise alone was presented, as $c$ is varied. This plotting procedure requires only that latencies be separated into two classes, those obtained from signal plus noise trials and those obtained from noise trials. However, data from different criterion settings are required. Since latency is a function of $|x-c|$, it is to be expected that median latency, given noise, say, would depend on $c$.

\section{The $R O C$}

Let $f(x)$ and $f(x-\mu)$ be the distributions of $X$ given noise and signal plus noise. Then $p_{H}(c)=R(c-\mu)$ and $p_{F A}(c)=R(c)$. The slope of the ROC is $f(c-\mu) / f(c) \equiv \beta$ (Green and Swets, 1966).

Theorem 5.1. If (i) $f(x)=f(-x)$, (ii) $f(x)$ tends to 0 as $R(x)$ tends to 0 and (iii) $-\log f(x)$ is convex,

\section{then either}

$$
\frac{1-p_{H}(c)}{p_{F A}(c)} \geqslant \beta \geqslant 1
$$

or

$$
\frac{1-p_{H}(c)}{p_{F A}(c)} \leqslant \beta \leqslant 1
$$

Proof. By Remark 2.5, $\beta$ is a monotonic function of $c$ if condition (iii) is satisfied. Therefore, $\beta \geqslant 1$ if and only if $c \geqslant \mu \mid 2$. If $c \geqslant \mu \mid 2, c>\mu-c$. Therefore, by Theorem 2.4, conditions (ii) and (iii) imply that

$$
\frac{f(c)}{R(c)} \geqslant \frac{f(\mu-c)}{R(\mu-c)}=\frac{f(c-\mu)}{F(c-\mu)}
$$

by condition (i).

Therefore,

$$
\frac{1-p_{H}(c)}{p_{F A}(c)}-\frac{F(c-\mu)}{R(c)} \geqslant \frac{f(c-\mu)}{f(c)}=\beta \geqslant 1 .
$$

Similarly for $c<\mu \mid 2$. 
This result was established for the normal distribution by Thomas and Legge (1970). For this distribution it can be strengthened.

CoRollary 5.2. If $f(x)$ is the normal density and if $\mu>2$, then the solutions of the inequality

$$
\frac{1-p_{H}(c)}{p_{F A}(c)} \geqslant \beta^{x}, \quad \beta \geqslant 1
$$

satisfy

$$
1 \leqslant x \leqslant \frac{\mu^{2}}{\mu^{2}-4} .
$$

Proof. The proof is established by noting that

$$
\left.\frac{d}{d \beta}\left[\frac{1-p_{H}(c)}{p_{F A}(c)}\right]\right|_{\beta=1}=\frac{f^{2}(\mu \mid 2)}{R(\mu \mid 2) f^{\prime}(\mu \mid 2)},
$$

and that, when $f(x)$ is normal, $f^{\prime}(x)=-x f(x)$ and, for $x>0$,

$$
f(x)\left(x^{-1}-x^{-3}\right)<R(x)<f(x) x^{-1} \quad \text { (Feller, 1962, p. 166). Q.E.D, }
$$

When a complete ROC is available, a test of the symmetry of $f(x)$ is to see whether the ROC is symmetric ahout the negative diagonal. By Remark 2.5 , a test of the convexity of $-\log f(x)$ is to see whether the slope of the ROC is monotonic (it should be remembered that response criterion is thought of as a value on a 'magnitude of sensation' axis, not on a likelihood ratio axis). Theorem 5.1 provides a test for the assumptions of symmetry and log-convexity, but this test can be applied even if a complete ROC is not available. One need have only a 'cluster' of two or more operating points $\left(p_{F A}(c), p_{H}(c)\right.$. Nuting that $\beta$ is the slope of the ROC, the test consists of comparing the empirical ROC slope with $\left[1-p_{H}(c)\right] / p_{F A}(c)$. The statistical properties of this test depend on the technique used in estimating the ROC, and will not concern us here.

It is interesting to note that condition (30) is not satisfied by ROCs that consist of two limbs, such as those generated by the threshold model of Luce (1963). To see this, let the equation of the lower limb of the symmetric ROC be

$$
y=K x, \quad 0 \leqslant x \leqslant \frac{1}{K+1}, \quad K>1 .
$$

Then, for

$$
\begin{gathered}
\frac{1}{2 K} \leqslant x \leqslant \frac{1}{K+1}, \\
K>\frac{1-y}{x}>1,
\end{gathered}
$$


i.e., the slope $(>1)$ of the ROC is greater than $\left(1-p_{H}\right) / p_{F A}$. Therefore, comparing the empirical ROC slope with $\left(1-p_{H}\right) / p_{F \cdot 4}$ is conceivably a good way of distinguishing between Luce's threshold theory and Signal Detection Theory.

\section{The $\mathrm{LOC}$}

Let us denote by $L_{\mu}(c)$ the typical latency on signal plus noise trials, and by $L_{0}(c)$ the typical latency on noise trials. Then $L_{\mu}(c)$ is a function of hit and miss latencies $t_{c-\mu}$ and $T_{c-\mu}$, and $L_{0}(c)$ is a function of false alarm and correct rejection latencies $t_{c}$ and $T_{c}$. If $c>\mu / 2$, from the inverse relation between latency and probability established earlier, it follows that $t_{c-\mu}<t_{c}$ and $T_{c-\mu}>T_{c}$, and it is not clear whether $L_{\mu}(c)$ is greater or less than $L_{0}(c)$. In this section we will show that the difference between $L_{u}(c)$ and $L_{0}(c)$ is a function of $c$ and that, for symmetric distributions, $L_{\mu}(c) \leqslant L_{0}(c)$ if and only if $c \leqslant \mu \mid 2$. In other words, for symmetric distributions if and only if the probability of a correct response, given signal plus noise, is greater than that of a correct response, given noise, (positive bias), then the latency on signal plus noise trials is less than the latency on noise trials.

Let $L_{\mu}(c)$ and $L_{0}(c)$ denote median latencies, and let $T$ denote the latency on a noise trial. Then

$$
\begin{aligned}
\operatorname{Prob}[T \leqslant \lambda(c)]= & \operatorname{Prob}(X \leqslant c) \operatorname{Prob}[X \leqslant c-x \mid X-c] \\
& +\operatorname{Prob}(X>c) \operatorname{Prob}[X>c+x \mid X>c] \\
= & F(c-x)+R(c+x) .
\end{aligned}
$$

Let $v_{c}$ be the solution of

$$
F(c-x)+R(c+x)=1 / 2
$$

then

$$
L_{0}(c)=\lambda\left(\nu_{c}\right)
$$

and

$$
L_{\mu}(c)=\lambda\left(v_{c-\mu}\right) .
$$

We have now to investigate the dependence of $\nu_{c}$ on $c$.

$$
h\left(c, v_{c}\right) \equiv F\left(c-v_{c}\right)+R\left(c+\nu_{c}\right)=1 / 2 .
$$

Therefore,

$$
\begin{aligned}
\frac{d \nu_{c}}{d c} & =-\frac{\partial h}{\partial c} / \frac{\partial h}{\partial \nu_{c}} \\
& =\frac{f\left(c-\nu_{c}\right)-f\left(c+\nu_{c}\right)}{f\left(c-\nu_{c}\right)+f\left(c+\nu_{c}\right)} .
\end{aligned}
$$


For simplicity, we consider the case where the range of $X$ is infinite and $f(x)=f(-x)$. Then $v_{c}$ decreases if $c<0$ and increases if $c>0$. Fig. 1 shows the LOC in this case. Since $\nu_{c}$ is a minimum when $c=0$, the maximum value of $L_{0}(c)$ and $L_{v}(c)$ is $\lambda\left(v_{c}\right)$. $L_{0}(c)=L_{\nu}(c)$ when $c=\mu \mid 2$ and $L_{0}(\mu \mid 2)$ is a decreasing function of $\mu$, since $\lambda\left(\mu_{c}\right)$ is decreasing for $c>0$. Finally, if and only if $c<\mu \mid 2, L_{0}(c) \geqslant L_{\mu}(c)$ and Prob(Hit) $\geqslant$ Prob(Correct Rejection), as asserted earlier.

This prediction was tested using the data of Carterette, et al. (see their Table I, 1965). Under the assumption that $f(x)=f(-x), c \leqslant \mu \mid 2$ if and only if $p_{H}+p_{F A} \geqslant 1$. Five of the six cases where $p_{H}+p_{F A}<1$ yielded $L_{\mu}(c)>L_{0}(c)$, and eleven of the twelve cases where $p_{H}+p_{F A}>1$ yielded $L_{\mu}(c)<L_{0}(c)$. Thus these data agree very well with the theory.

\section{Concluding Comments}

This paper has attempted to relate the observable latency in a signal detection experiment to a detection model by considering, on the one hand, properties of the detection model and, on the other, properties of an unobservable, elemental latency. The detection model considered is similar to that of Signal Detection Theory, except that the underlying random variable $X$ is not assumed to be a likelihood ratio. The distributions of $X$ given noise and signal plus noise are assumed to have the same shape, $f(x)$ and $f(x-\mu)$, respectively, so that the properties of $f(x)$ are a sufficient description of the detection model. The unobservable latency is the time taken to determine whether or not $X$ exceeds a criterion $c$ and is given by $\lambda(|X-c|)$, a function of $|X-c|$ only. The sign of the slope and of the curvature of $\lambda(x)$ are the two properties of this latency which we have considered.

Observable latency can be analysed in many ways in order to test its consistency with theory, and we have selected only a few of these ways. For most of the paper we considered latency conditional on signal and on response, e.g. false alarm latency, and in the previous section we considered latency, conditional on signal only. For latency conditional on signal and response, we considered first order properties, namely, the slope of the latencyprobability curve, and second order properties, namely the slope of a latency differenceprobability curve, and that of a latency dispersion-probability curve.

The main result on first order properties of latency is that (i) if $-\log f(x)$ is convex and if $\lambda(x)$ is decreasing then the latency of a response decreases as the probability of the response increases (Theorem 2.12). Neither $f(x)$ nor $\lambda(x)$ can be observed, but it is possible to derive some of their properties by considering response probabilities and ignoring latencies, and by considering latencies and ignoring response probabilities. The ROC and LOC, respectively, are useful statistics for such an analysis because

(ii) the slope of the ROC is monotonic if and only if $-\log f(x)$ is convex (Remark 2.5), 
(iii) if $f(x)=f(-x)$ and if $-\log f(x)$ is convex then the slope (Theorem 5.1) of the $\mathrm{ROC}$ at the point $(u, v)$ lies between 1 and $(1-v) / u$,

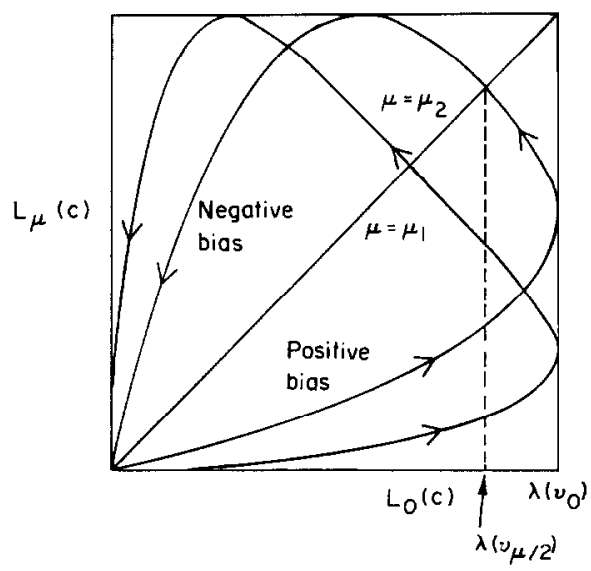

FIG. 2. The Latency Operating Characteristic for symmetric distributions $f(x)$ and $f(x-\mu)$, and two values of $\mu, \mu_{1}$ and $\mu_{2}\left(<\mu_{1}\right)$.

(iv) if $\lambda(x)$ is decreasing then the LOC isa closed loop (Fig. 2). Therefore, it is suggested that the ROC and the LOC should be plotted and used as crude checks of the assumptions of the detection and latency models, before the relationship between data and theory is assessed. The main results on second order properties of latency are [assuming $-\log f(x)$ is convex and $\lambda(x)$ is decreasing]:

(v) if $f(x) \mid f^{\prime}(x)(x>a)$ is convex, and if the difference between false alarm and hit latencies decreases as hit latency decreases, then $\lambda(x)$ is convex (Theorem 3.9) and

(vi) if dispersion of latency is directly related to typical latency then $\lambda(x)$ is convex (Theorem 3.10 a).

The distribution of observable latency $p_{c}(t)$ is that of a transformed random variable, and we have determined what properties this transformation $\lambda(x)$, must have for the latency distribution to have certain stated properties. Assuming that the range of $X$ is infinite, we have found that

(vii) $p_{c}(t)$ is continuous at $t=0$ only if $\lambda(x) \neq 0$ for all finite $x$.

(viii) $\lim _{t \rightarrow 0} p_{c}(t)=0$ if $\lim _{x \rightarrow \infty} x^{b+1} f(c+x)=0$, where $\lambda(x)=x^{-b}, b>0$,

(ix) $p_{c}(t) 0$ for all finite $t$ only if $\lim _{x \rightarrow 0} \lambda(x)=\infty$,

The problem of accounting for latency distributions with 'high' tails has been considered by McGill and Gibbon (1965) who proposed the general gamma distribution, and by Snodgrass, Luce and Galanter (1967), who proposed the double monomial. 
In the present context, the high tail arises fairly naturally because of the interpretation of latency as a transformed random variable.

The proofs of many of the results in this paper depends on the fundamental Lemma 2.3. This Lemma is a tool for establishing inequalities involving $F(x)$ and $1-F(x)$, as was illustrated in Theorems 2.4 and 3.5 , and, therefore, it should be useful for similar analyses of detection-latency models which give rise to truncated distributions.

\section{ACKNOWLEDGMENTS}

I am greatly indebted to Jerome L. Myers for suggesting this problem and for many stimulating discussions, and to the referees for their comments. Most of the work presented here was done while the author was at University College, London, support having been obtained from a Science Research Council (U. K.) grant.

\section{REFERENCES}

Audley, R. J., AND Mercer, A. The relation between decision time and relative response frequency in a blue-green discrimination. British Journal of Mathematical and Statistical Psychology, 1968, 21, 183-192.

Barlow, R. E., Marshall, A. W., and Proschan, F. Properties of probability distributions with monotone hazard rate. Annals of Mathematical Statistics, 1963, 34, 375-389.

CARTERETTE, E. C. Random walk models for reaction times in signal detection and recognition. Proceedings of the XVIIIth Congress of Psychology (Moscow), 1966, 16, 84-95.

Carterette, E. C., Friedman, M. P., and Cosmides, R. Reaction-time distributions in the detection of weak signals in noise. Journal of the Acoustical Society of America, 1965, 38, 531-542.

FELler, W. An introduction to probability theory, Vol. 1. New York: Wiley, 1962.

GrEEN, D. M., AND LuCE, R. D. Detection of auditory signals presented at random times. Perception and Psychophysics, 1967, 2, 441-449.

Green, D. M., AND Swets, J. A. Signal detection theory and psychophysics. New York: Wiley, 1966.

LAMing, D. R. J. From choice-reaction times to psychophysics. 1969. Unpublished manuscript.

Lehmann, E. L. Testing statistical hypotheses. New York: Wiley, 1959.

LuCE, R. D. A threshold theory for simple detection experiments. Psychological Review, 1963, 70, 61-79.

MCGill, W. J., AND GtBBon, J. The general-gamma distribution and reaction times. Journal of mathematical Psychology, 1965, 2, 1-18.

Moss, S. M., Myers, J. L., ANd Filmore, T. Short-term recognition memory of tones. Perception and Psychophysics, 1970.

Myers, J. L., AND Thomas, E. A. C. Implications of latency data for continuous and discrete state models of signal detection, to appear.

Norman, D. A., AND Wickelgren, W. Strength theory of decision rules and latency in retrieval from short-term memory. Journal of Mathematical Psychology, 1969, 6, 192-208.

Snograss, J. G., Luce, R. D., And Galanter, E. Some experiments on simple and choice reaction time. Journal of Experimental Psychology, 1967, 75, 1-17. 
Sмiтн, P. T. Cost, discriminability and response bias. British Journal of Mathematical and Statistical Psychology, 1968, 21, 35-60.

Thomas, E. A. C. Reaction-time studies: the anticipation and interaction of responses. Ibid, $1967,20,1-29$.

Thomas, E. A. C., And Legge, D. Probability matching as a basis for detection and recognition decisions. Psychological Review, 1970, 77, 65-72. 